Piecemeal-EMR (p-EMR) was longer than en-bloc EMR (mean duration $=63$ vs 48 min, mean difference $=14$ min, $\mathrm{p}$ $<0.0022$.

Sigmoidoscopy EMR $(\mathrm{n}=52)$ was not significantly longer than colonoscopy EMR $(\mathrm{n}=43)$ (mean times: 53 vs $50 \mathrm{~min}$ mean difference $=3 \min \mathrm{p}=0.28)$.

Removal of $>1$ polyps $(n=74)$ was not significantly longer than that for 1 polyp $(\mathrm{n}=21$ ) (Mean time: 53 vs 46 min, mean difference $=7 \min \mathrm{p}=0.1115$ ).

Conclusion The time taken for endoscopic mucosal resection of colonic polyps did not exceed the allocated significantly. The factors that affected the duration significantly were age of the patient and size of the polyp. Piecemeal EMR was also associated with longer duration than enbloc- EMR.

These factors should be taken into consideration when booking patients for planned endoscopic resection of colonic polyps. Disclosure of Interest None Declared.

\section{PTH-055 USS SMALL BOWEL FOR CROHN'S DISEASE - SINGLE CENTRE EXPERIENCE IN MEDIUM SIZED DGH}

M Usman-Saeed*, T Skouras, T Patani, J Whalley, S Khalid. Warrington and Halton Hospital, Warrington, UK

\subsection{6/gutjnl-2014-307263.50}

Introduction USS (Ultra sound scan) in experienced hands is good radiological modality in diagnosing, confirming or ruling out Crohn's disease. USS is easy, cost effective and without risk of radiation as compared to CT (Computerised tomography) or MRI (Magnetic resonance imaging) scans. USS can be particularly useful in younger population with Crohn's who may require repeated imaging.

We were interested to look at outcomes of USS of small bowel performed by dedicated GI (gastrointestinal) radiologist in our hospital.

Methods We audited efficacy of USS in patients with known, suspected or to exclude Crohn's disease. A retrospective review of patient records using specialised electronic Medicorr and PACS databases was performed. Demographic information, diagnosis, procedural details and subsequent result of USS was entered into a Microsoft Access database and analysed using Microsoft Excel.

Results A total of 145 patients underwent USS of small bowel in 18 months. The average age was 40 . There were 97 females and 48 males. USS was performed in fifty seven patients with known, twenty four suspected and in 64 patients to exclude Crohn's disease. In known disease group thirty four (60\%) had normal USS (these patients were in remission), and twenty three (40\%) had USS findings consistent with active, small or large bowel disease, strictures, fistulating disease and abscesses.

For suspected group, 17 patients (70\%) had normal and seven $(30 \%)$ had features consistent with Crohn's. Five of these positive scan patients had MRI scans. 2 MRI scans confirmed USS findings, 3 were normal and required endoscopic confirmation.

In exclusion group 64 had USS with 61 (95\%) normal and 3 (4\%) abnormal. Findings of 3 abnormal scan showed gallbladder polyp, caecal thickening (colonoscopy confirmed caecal tumour) and non- specific ileitis with negative colonoscopy and histology. Conclusion Our results show that USS is reliable and as good as other imaging if done by experienced radiologist with special interest in GI radiology. USS is cost effective, readily available and free of radiation. It is cheap, portable, flexible and user- and patient-friendly. It can save cost and time required for CT and MRI scan especially in district general hospital.
We recommend utilising USS small bowel to assess disease activity in known cases and also to confirm or exclude the disease provided experienced GI radiologist is available.

\section{REFERENCES}

1 Nylund $\mathrm{K}$, Ødegaard $\mathrm{S}$ et al. Sonography of the small intestine. World I Gastroenterol 2009:15(11):1319-1330

2 Parente F, Greco $\mathrm{S}$ et al. Modern Imaging of crohn's disease using bowel ultrasound. Inflamm Bowel Dis 2004; 10(4):452-461

Disclosure of Interest None Declared.

\section{PTH-056 PRODUCTIVE ENDOSCOPY PROJECT}

${ }^{1} \mathrm{~N}$ Taggart ${ }^{*},{ }^{2} \mathrm{~J}$ Lucking, ${ }^{1} \mathrm{C}$ Eccles, ${ }^{1} \mathrm{C}$ Cooper, ${ }^{1} \mathrm{~K}$ Kennedy, ${ }^{1} \mathrm{D}$ Daw, ${ }^{1} \mathrm{~L}$ Williams, 'W Hymer, 'D Mcguinness, 'L Flavell, '1 Dahill, 'S Sarkar. 'Endoscopy, Royal Liverpool Hospital, Liverpool, UK; ${ }^{2}$ NHS IQ, NHS UK, London, UK

\subsection{6/gutjnl-2014-307263.502}

Introduction In June 2012 the Royal Liverpool endoscopy department was chosen by NHS Improving Quality to be a pilot site for the development and implementation of a productive endoscopy toolkit. The outcome was improved efficiency, safety, patient experience and team-working.

Methods The aim was to apply processes and cultures of lean thinking to endoscopy and complete a series of modules that increase safety, reliability of care, improve team performance and improve efficiency.

A six month "diagnostic phase" involved collecting data on all aspects of the service to identify areas for improvement. A number of modules were completed that tackled the inefficiencies identified and involved the engagement of all staff groups.

Results Stock: The "well organised unit" module identified $£ 7,500$ of redundant stock. Clearing the extra space meant equipment could be re-allocated, releasing two bed spaces in recovery.

Enemas: $44 \%$ of patients were not compliant with their enemas. Of the $56 \%$ who were complaint $14 \%$ was ineffective. This impacted on the daily running of the department due to unprepped patients. This has led to a different type of enema now being used along with the development of more detailed patient information.

Department efficiency: Start/ Stop audit identified that 15\% of the time, rooms were not utilised and $85 \%$ of time the list started late, due to staff or the room not being ready for the start of the morning session. Sharing this information with the teams led to more motivation and demonstrable leadership by senior Clinicians and Nursing staff to improve workforce compliance. $50 \%$ of lists finished late, $43 \%$ was due to complex procedures over-running this has led to stricter vetting and more points allocated for these procedures. Further data analysis has shown that Thursday and Friday run late $65 \%$ of the time due to endoscopists arriving late for the start of the afternoon session because of over-run clinics. This has led to a review of the scheduling template.

Patient experience: Long waits were problematic for patients particularly, time after admission, prior to procedure, patients waited 12 to 90 mins and time spent post procedure, waiting for discharge, up to 210 mins. Discharges were also slower in the afternoon than in the evening. Review of skill mix and assigning specific roles to discharge will help to expedite effective and timely discharge.

Conclusion Working through the innovative tools and processes of each module has allowed us to use an evidence based methodology for quality improvement. The use of advanced data 
collection has highlighted clearly what improvements are needed based upon facts and not assumptions.

Disclosure of Interest None Declared.

\section{PTH-057 A STUDY OF NON-RESPONDENTS' PERSPECTIVES OF THE BARRIERS AFFECTING BOWEL CANCER SCREENING UPTAKE IN WALES FOR THE PURPOSES OF INFORMING PROGRAMME DELIVERY AND MANAGEMENT OF SCREENING}

${ }^{1} \mathrm{~N}$ Avery*, ${ }^{2} \mathrm{~S}$ Darwin. 'Directorate for Public Health, Welsh Government, Cardiff, UK; ${ }^{2}$ Bowel Screening UK, Public Health Wales, Llantrisant, UK

10.1136/gutjnl-2014-307263.503

Introduction Decreasing participation levels in the Welsh bowel screening programme are cause for concern for Bowel Screening Wales (BSW) and the Welsh Government. BSW, the population screening programme was launched in 2008 and was the first home-testing screening programme in Wales. The programme has successfully detected almost 700 cancers and identified a significant number of polyps for removal that may have otherwise developed into cancer. Despite this, participation in the programme has declined and was 52\% during the last quarter of 2011/2012.

Methods The research aimed to seek the views of those who have not responded to their latest invitation to participate in bowel cancer screening (BCS). Methodology for the study included development of a questionnaire based around behavioural theories and qualitative interviews. The research was conducted using a mixed-methods design with a postal questionnaire to BCS non-respondents (quantitative) and semistructured interviews with non-respondents and screening professionals (qualitative). These methods probed into attitudes and beliefs to find out to what extent these traits influenced behaviour. The research was also combined with a literature review.

The questionnaires were randomly sent to 988 participants with a 3 week deadline given for completion and return. In total 101 questionnaires were returned, 9 were not useable either because the individuals had other arrangements in place (like private healthcare) or declined to participate.

7 semi-structured telephone interviews were also undertaken with responders to the questionnaire who agreed to contact, together with service providers. The data from questionnaires was analysed for emerging themes and issues.

Results Findings show a wide range of factors preventing participation and that a 'one size fits all' approach to increasing uptake is unlikely to work. The evidence from primary and secondary research points to tailored interventions being a more effective approach. The findings from the questionnaire indicated a reasonable level of knowledge about the disease and its signs and symptoms. However, when tested during interviews, the responses showed that awareness levels were poor. Qualitative responses suggested that upfront key messages about the diseases prevalence and the benefits of early detection were needed.

Conclusion Based on the research undertaken, the following recommendations were made to increase the uptake of the screening programme: Raise levels of awareness of bowel cancer and the screening programme; review information to ensure upfront key messages and have sufficient information about the balance of harms and benefits to make an informed choice: pilot a range of small-scale process changes, particularly to the reminder processes.

Disclosure of Interest None Declared.
PTH-058 A CENTRES EXPERIENCE OF SETTING UP A NEW BOWEL CANCER SCREENING CENTRE AND PREPARING FOR BOWEL SCOPE

${ }^{1} \mathrm{~N}$ Taggart*, ${ }^{1} \mathrm{~J}$ Batterton, ${ }^{1} \mathrm{D}$ Mcguinness, ${ }^{1} \mathrm{G}$ Fitzgerald, ${ }^{1} \mathrm{~B}$ Morton, ${ }^{1} \mathrm{P}$ Otoole, ${ }^{1} \mathrm{~S}$ Subrumanian, ${ }^{1} \mathrm{~N}$ Haslam, ${ }^{1,2} \mathrm{~B}$ Oates, 'S Sarkar. 'Endoscopy, Liverpool and Wirral Bowel Screening, Liverpool, UK; ${ }^{2}$ Endoscopy, Arrowe Park Hospital, Liverpool, UK

\subsection{6/gutjnl-2014-307263.504}

Introduction The population served by the Merseyside and North Cheshire Programme was 1.7 million based at University Hospitals Aintree was asked to split because of the imminent introduction of Bowel scope. Authorisation for forming of new centre at the Royal Liverpool Hospital (RLH) by central office was granted in the summer 2013 and implementation was required by February 2014. Methods The Liverpool and Wirral Bowel screening centre (LandW BSC) was formed with a $45 \%$ share of the in the population. The service was designed with the aim to run the FOBT service and then introduce the Bowel Scope (BS) as second wave for the serving population of 802,000.

Results Workforce: The current workforce at the Royal Liverpool (RLH) included 4x BCSP screening colonoscopists of which one became Clinical Director of the centre. The endoscopy service manager supportive for the nursing and operational aspects and the Endoscopy Admin manager supported the administration aspect. There was full managerial backing by the directorate manager. In addition, programme manager, Lead SSP, 3x New SSPs, $4 \mathrm{x}$ admin staff were appointed.

Collaborations and Engagement: The logistical issues and training of the new SSPs and Admin staff was heavily facilitated by the current Merseyside and Cheshire programme. Engagement and support also included the National Team, CCG, Local QA team and Screening and Immunisation Regional Team. Implementation group representation from all parties. A business was supported by the trust board. The strong collaboration was made with the team from Arrowe Park Hospital (APH).

BowelScope: Stategically, new urology centre had spare capacity for 8 lists to include some evenings and weekends sessions at Broadgreen Hospital was identified as good access site for Liverpool. For the Wirral site, APH was identified as the site to deliver bowelscope and for the population would require 4 lists. Eight endoscopists were further identified (6 nurse endoscopists, 1 Fellow and 1 radiographer-also programme manager) as needing accreditation for bowelscope. All mentees were assigned a mentor ( 3 BCSP colonoscopists) and put on the preaccreditation course at RLH. Exams were planned over 3, UKdays for all the endoscopists in April 2014. Prior to the exam, all endoscopists, had 2 flexi lists booked with their mentors at RLH as well as ad-hoc sessions to help with MCQ.

Outcome: The LandW BCSC got approval by national office in Jan 2014 with a go live date in Feb to take over FOBT service. Bowelscope is planned for September 2014.

Conclusion The setting up of a new centre in such a short period of time was extremely challenging but successful due to good team work, planning and collaboration.

Disclosure of Interest None Declared.

\section{PTH-059 A MODEL DESIGNED TO CALCULATE THE COST OF CARE FOR INFLAMMATORY BOWEL DISEASE (IBD).}

${ }^{1} \mathrm{~N}$. Ghosh*, ${ }^{2} \mathrm{~S}$. Leonard, ${ }^{2} \mathrm{G}$. Miles, ${ }^{1} \mathrm{P}$. Premchand. ${ }^{1}$ Barking, Havering and Redbridge University Hospitals NHS Trust, Queen's Hospital, Romford, UK; ${ }^{2}$ Costello Medical Consulting, Cambridge, UK

10.1136/gutjnl-2014-307263.505 